\title{
Combined transcranial direct current stimulation and robotic upper limb therapy improves upper limb function in an adult with cerebral palsy
}

\author{
Kathleen M. Friel ${ }^{\mathrm{a}, \mathrm{b}, \mathrm{c}, *}$, Peter Lee ${ }^{\mathrm{a}, \mathrm{d}}$, Lindsey V. Soles ${ }^{\mathrm{a}, \mathrm{c}}$, Ana R.P. Smorenburg ${ }^{\mathrm{a}}$, \\ Hsing-Ching Kuo ${ }^{\mathrm{a}, \mathrm{f}}$, Disha Gupta ${ }^{\mathrm{a}, \mathrm{b}, \mathrm{e}, \mathrm{i},}$ and Dylan J. Edwards ${ }^{\mathrm{a}, \mathrm{g}, \mathrm{h}}$

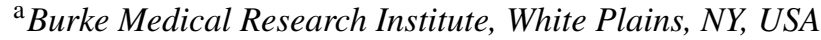 \\ ${ }^{\mathrm{b}}$ Brain Mind Research Institute, Weill Cornell Medical Center, New York, NY, USA \\ 'Blythedale Children's Hospital, Valhalla, NY, USA \\ ${ }^{\mathrm{d}}$ Montefiore Medical Center, Bronx, NY, USA \\ ${ }^{\mathrm{e}}$ Department of Computational Science and Engineering, Cornell University, Ithaca, NY, USA \\ ${ }^{\mathrm{f}}$ University of Calgary, Alberta Children's Hospital Research Institute, Calgary, Alberta, Canada \\ ${ }^{\mathrm{g}}$ Department of Neurology, Weill Cornell Medical Center, New York, NY, USA \\ ${ }^{\mathrm{h}}$ School of Medical and Health Sciences, ECU Australia \\ ${ }^{\mathrm{i}}$ Helen Hayes Hospital, West Haverstraw, NY, USA
}

\begin{abstract}
.
BACKGROUND: Robotic therapy can improve upper limb function in hemiparesis. Excitatory transcranial direct current stimulation (tDCS) can prime brain motor circuits before therapy.

OBJECTIVE: We tested safety and efficacy of tDCS plus robotic therapy in an adult with unilateral spastic cerebral palsy (USCP).

METHODS: In each of 36 sessions, anodal tDCS $(2 \mathrm{~mA}, 20 \mathrm{~min})$ was applied over the motor map of the affected hand. Immediately after tDCS, the participant completed robotic therapy, using the shoulder, elbow, and wrist (MIT Manus). The participant sat in a padded chair with affected arm abducted, forearm supported, and hand grasping the robot handle. The participant controlled the robot arm with his affected arm to move a cursor from the center of a circle to each of eight targets (960 movements). Motor function was tested before, after, and six months after therapy with the Wolf Motor Function Test (WMFT) and Fugl-Meyer (FM).

RESULTS: Reaching accuracy on the robot task improved significantly after therapy. The WMFT and FM improved clinically meaningful amounts after therapy. The motor map of the affected hand expanded after therapy. Improvements were maintained six months after therapy.

CONCLUSIONS: Combined tDCS and robotics safely improved upper limb function in an adult with USCP.
\end{abstract}

Keywords: Neuromodulation, neuroplasticity, rehabilitation

\section{Introduction}

*Address for correspondence: Kathleen Friel, Ph.D., Burke Medical Research Institute, 785 Mamaroneck Avenue, White Plains, NY 10605, USA. Tel.: +1 914368 3116; Fax: +1 914597 2225; E-mail: kaf3001@med.cornell.edu.
Cerebral palsy $(\mathrm{CP})$ is the most common pediatriconset neurological disorder affecting movement. CP is caused by a nonprogressive brain injury or stroke before birth or during early life. People with unilateral 
spastic cerebral palsy (USCP), the most common subtype, have movement impairments primarily on one side of the body. Importantly, motor deficits can have profound, permanent consequences on quality of life and independence, particularly in adults. Few therapies exist for upper limb rehabilitation in adults with USCP. The goal of this pilot study was to determine feasibility and efficacy of combined upper limb robotic therapy and excitatory transcranial direct current stimulation (tDCS) for adults with USCP. Here we preset a case study of one participant. We examined changes in robotic task performance, clinical motor outcomes, and motor map neurophysiology after 36 sessions of tDCS plus upper limb robotic therapy.

Motor rehabilitation that follows the basic principles of motor learning appears to be optimal for recovery (Kitago et al., 2013). This requires repeated practice sessions that are sufficiently distributed to allow memory consolidation (Censor, 2013), resulting in a higher state of functional performance. Robotic therapy is an effective method to facilitate this in chronic hemiparesis (Turner et al., 2013). Robotic therapy devices can sense the amount of force a patient produces in a functional motor task and adjust the amount of assistance accordingly, such that an independently non-attainable functional goal is achieved (Aarts et al., 2010). There is mounting evidence that robotic therapy leads to a sustained positive effect on movement function in chronic stroke (Norouzi-Gheidari et al., 2012). Movements trained in robotic therapy translate to functional outcome measures in patients with hemiparesis (Bosecker et al., 2010).

TDCS modulates the excitability of a targeted brain region non-invasively by altering neuronal membrane potentials (Paulus, 2004). TDCS can prime brain motor circuits before robotic therapy. This innovative combination of two emerging technologies improves upper limb function in adult stroke survivors (Giacobbe et al., 2013; Liew et al., 2014). Since USCP has a similar clinical presentation as hemiparetic stroke, we hypothesized that this therapy can also improve upper limb motor skill in adults with USCP.

A key difference between adult-onset stroke and pediatric-onset USCP is the organization of the motor system. In adult stroke, the contralateral wiring of the motor system has been established, but pediatric USCP disrupts motor system development. In as many as $30 \%$ of people with USCP, the contralesional hemisphere controls movement of both hands, controlling the impaired hand via ipsilateral connections
(Staudt, 2007; Staudt et al., 2004). We have shown these ipsilateral connections to be adaptive and plastic (Friel et al., 2016; Smorenburg et al., 2016). Since the wiring of the motor system is variable among people with USCP, in this study we used single-pulse transcranial magnetic stimulation (TMS) to first identify the locus of control of the impaired hand, then we targeted tDCS to stimulate this location. This personalized, targeted approach is hypothesized to optimize recovery for an individual.

\section{Methods}

The study participant was a 37 year old male who acquired USCP at age 18 months due to complications from a motor vehicle accident. The left side of his body is impaired. This study was approved by the Burke Rehabilitation Hospital Institutional Review Board. The participant provided written informed consent for all study procedures.

We conducted a battery of assessments: 1) Clinical outcome measures - the Wolf Motor Function Test, the Upper Limb Fugl-Meyer, and grip strength of the affected hand; 2) kinematic movement assessments on the robot; and 3) brain motor mapping with single-pulse transcranial magnetic stimulation (TMS). Assessments are described below.

\subsection{Clinical outcome measures}

Clinical outcome measures were taken before the first day of the intervention, immediately after the final day of the intervention, and six months later. The Fugl-Meyer is a validated and reliable evaluation instrument used for measuring performance-based impairment in stroke patients (Dong et al., 2013; Gladstone et al., 2002; Malouin et al., 1994). The Wolf Motor Function Test assesses upper extremity function by asking the participant to complete 15 motor-based tasks and 2 strength-based tasks (Morris et al., 2001). Grip strength was assessed using a Jamar hydraulic hand dynamometer (Lafayette Instrument, Lafayette, IN). The average of three trials was computed.

\subsection{Upper limb robotic assessments and therapy}

We used In Motion Technology (IMT; Massachusetts Institute of Technology) planar and wrist robots in this study (Volpe et al., 2009). For both assessments and training, the participant sat in a 
foam-padded chair facing the robot and a video screen. The impaired arm was abducted, forearm supported, and hand lightly grasping the robot handle, and velcro straps lightly held the forearm and fingers secure. A cursor on the video screen displayed the targets and tracked the movement of the patient's upper limb.

\subsection{Robotic evaluations}

The participant performed unassisted movements toward targets presented in 8 positions equally spaced around a 14-cm radius circle ("star reaches") or around an ellipse with major axis corresponding to wrist flexion/extension of 22.5 degrees and minor axis corresponding to radial/ulnar deviation of 12.5 degrees ("circle drawing") (Krebs et al., 1999). Targets were presented in clockwise order similar to a robot-assisted therapy session (see below). A total of 80 movements of star reaches (done on both the planar and wrist robots) and 20 circle drawings (done only on the planar robot) were performed in each evaluation session.

Robotic evaluations (star reaches plus circle drawing) were done on each arm before the first day of the intervention, midway through the intervention, immediately after the final day of the intervention, and six months later. Additional circle drawing evaluations were done using the impaired arm at weeks 3 , 5 , and 10 of therapy. These were done to track transfer of skill from the trained task (star reaches) to an untrained task (circle drawing).

\subsection{Robotic therapy}

The participant received a total of 36 sessions comprising 1 hour of interactive robotic training, using the shoulder-elbow (planar) MIT Manus robot (30 mins), followed by the wrist robot (30 mins). The interactive robot features have been discussed at length elsewhere (Krebs et al., 1999) and involve visuomotor tasks, moving the robotic manipulandum according to targets on a computer screen mounted at eye level. A key feature of MIT robots is the low near isotropic inertia and reduced friction in the robot arm so that, when appropriate, it can "get out of the way." The force required to move the robotic arm is minimal, comparable to moving unrestricted, and if a patient cannot move the robot arm, it will guide the limb to provide an adaptive sensorimotor experience.

The participant alternated using the planar and wrist robots at each therapy session. Thus, the participant completed 18 training session on the planar robot and 18 sessions on the wrist robot. At the beginning of each session, the participant completed five rounds of target reaching without the assistance of the robot, followed by three blocks of 320 trials in which the robot assisted movement as needed. After each block, the participant completed five rounds of target reaching without the assistance of the robot. Thus, each session consisted of 64 unassisted reaches and 960 assisted reaches.

\subsection{Robotic kinematic measures}

We used a set of MATLAB programs (The Mathworks Inc., Waltham, MA) to extract measures of reach accuracy and movement smoothness (ratio of mean speed to peak speed) for each session. For star reaches, we measured the distance between the endpoint of the participant's reach and the location of the intended target. We also measured smoothness of reaching toward each target. We calculated overall reach error and smoothness, as well as reach error and smoothness in each reach direction.

For circle drawing, we measured circularity of drawn ellipses, the ratio of the length of the major axis to minor axis. For a perfectly round circle, circularity would equal 1.

\subsection{TMS motor mapping}

TMS motor mapping was done before the first day of the intervention and immediately after the final day of the intervention. Single-pulse TMS was conducted with a MagStim 200 and a $70 \mathrm{~mm}$ Figure-of-Eight stimulating coil (MagStim, Dyfed, United Kingdom). During TMS, muscle activity was recorded using surface electromyography (EMG) electrodes. A multi-channel recording system (NeuroConn, Germany) simultaneously recorded EMG activity bilaterally in the first dorsal interosseous (FDI) and extensor carpi radialis (ECR) muscles. These muscles were selected to yield a motor map with proximal and distal musculature.

The TMS device triggered the recording system, such that EMG activity was recorded at $4000 \mathrm{~Hz}$ $400 \mathrm{~ms}$ before and $400 \mathrm{~ms}$ after each TMS pulse is delivered. The position of each stimulation point over the scalp was recorded in xyz coordinates and overlaid on a standard MRI using neuronavigation software (Brainsight, Rogue Resolutions, Montreal, Canada). 
To ensure all representations of the hand were found, stimulation began in a medial portion of the affected (right side) primary motor cortex (M1). The coil was moved laterally to search for a motor evoked potential (MEP) in the affected (left) FDI. In this participant, there was no MEP in the affected M1, which is common in people with USCP. We then stimulated the contralesional (left) M1, and found MEPs of both the left and right FDIs. We determined the resting motor threshold (rMT), which is the minimum stimulator output required to evoke an MEP over $50 \mu \mathrm{V}$ in the FDI muscle in 6 of 10 trials in relaxed muscles (Kleim et al., 2007).

We superimposed a circular grid of $1 \mathrm{~cm}$-spaced sites, radius $10 \mathrm{~cm}$ on the MRI (in Brainsight). The grid was centered around the point of strongest activation of the affected FDI ("hotspot"). The coil was used to deliver 2-3 TMS pulses at an intensity of $110 \%$ rMT at each grid point until MEPs were no longer found.

We processed MEP data by exporting EMG to MATLAB for filtering and processing in a customwritten analysis program. The MEP for each muscle at each stimulation site was defined as the peak-topeak amplitude of the EMG response. Trials were excluded if the participant was not relaxed before the TMS pulse. MEPs will be averaged for stimuli delivered at the same site. If the average MEP is greater than $50 \mu \mathrm{V}$ for a muscle at a site, that site was categorized as controlling that muscle. Total area enclosing digit and wrist sites for each hand was measured.

\subsection{Colocalization of tDCS and motor map of affected hand}

Before each therapy session, the participant received $20 \mathrm{~min}$ of $2 \mathrm{~mA}$ excitatory (anodal) tDCS (Soterix $1 \times 1$ Limited Total Energy device, New York, NY). We placed the excitatory sponge over the location of the motor map of the impaired hand, which was located with single-pulse TMS. The anodal sponge was placed on the contralateral forehead. Both sponges were moistened with saline and held in place with a headband. Before and after stimulation, we asked the participants to rate the presence of any symptoms related to tDCS (headache, tingling, itching).

\subsection{Statistics}

For clinical outcomes and TMS measures, statistical differences could not be evaluated in this single-participant case study. For robotic kinematics, since many trials were done over the 36 days of training and four days of assessments, it was possible to examine statistical differences. We used repeated measures ANOVA with Bonferronicorrected post-hoc comparisons to evaluate differences in kinematics over the intervention.

\section{Results}

In this pilot study, we found that combined tDCS and robotic upper limb therapy can safely improve upper limb function in adult USCP.

\subsection{Improvements in clinical outcomes}

Clinical outcomes are summarized in Table 1. After the intervention, all measures improved. The Wolf improved 7.0 points, which is a clinically meaningful change, while the Fugl-Meyer improvements of 3.0 pointes neared a clinically meaningful change (Clinically meaningful change: Fugl-Meyer 4.25 points (Page et al., 2012), Wolf 4.36 points (Lin et al., 2009)). Improvements were maintained six months after intervention.

\subsection{Improvements in kinematic measures}

We examined movement smoothness and reaching error of robotic assessments and training, since these outcomes have been most responsive to change and correlated with clinical outcomes (Edwards et al., 2012). Figure 1 shows representative example movement trials for the planar and wrist robot star reaches and planar robot circle drawing.

Over the course of the intervention, there was no significant change in movement smoothness on the

Table 1

Changes in clinical outcome measures after therapy

\begin{tabular}{lccc}
\hline Outcome Measures & Before Therapy & After Therapy & Six Months After Therapy \\
\hline Wolf Motor Function Test & 45 & 52 & 52 \\
Fugl-Meyer Upper Limb & 10 & 13 & 13 \\
Grip Strength (kg) & 9.5 & 10.4 & 10.8 \\
\hline
\end{tabular}




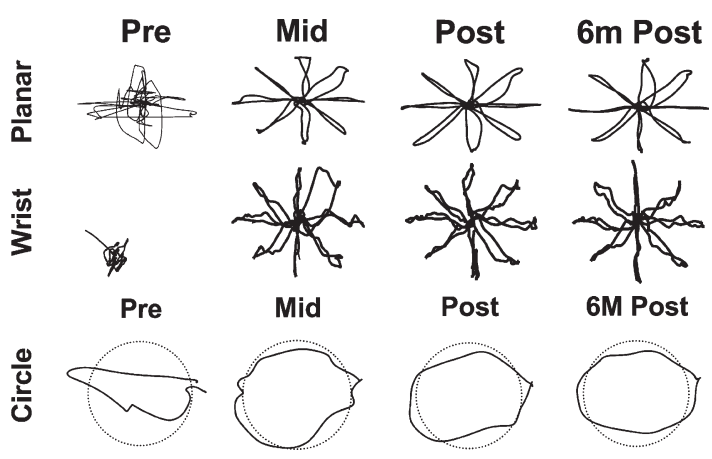

Fig. 1. Representative traces of movement trials on the planar robot star reach task (top panel), wrist robot star reach task (middle panel), and planar robot circle drawing task (botton panel).

planar (Fig. 2A) or wrist (Fig. 2B) robot. In contrast, reaching accuracy on the planar (Fig. 2C) and wrist (Fig. 2D) robots improved significantly. During the first half of the intervention (pre to mid), there was a statistically significant decrease in reach error on the planar robot $(\mathrm{F}(3,126)=34.6, p<0.0001)$ and on the wrist robot $(\mathrm{F}(3,126)=55.0, p<0.0001)$. Beyond the midpoint of the intervention, there was no further improvement in reach error $(p>0.05)$.

We noted that reach error may be different depending on the direction of reach. Therefore, we examined reach error in each of the eight task directions (Figs. 3 and 4). For the planar robotic task (Fig. 3), reach error was significantly elevated in the top and right

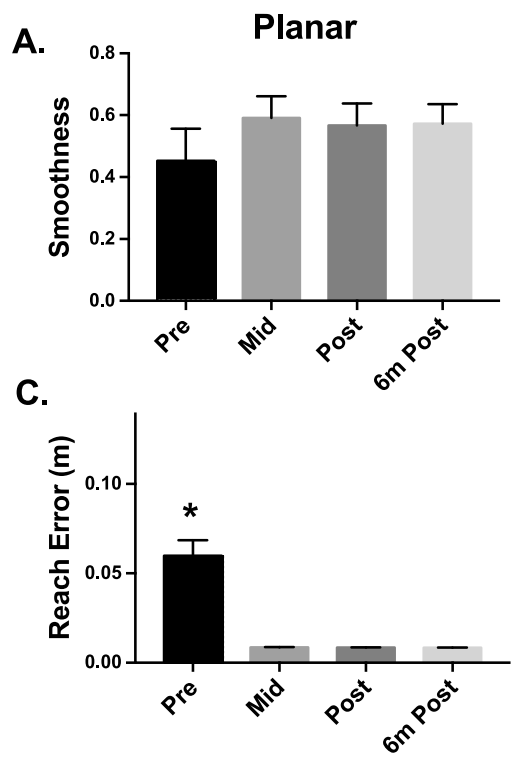

directions $(\mathrm{F}(3,16)>5.0, p<0.0001)$, indicating that the participant had most difficulty extending his left (impaired) arm. For the wrist robotic task (Fig. 4), reach error was similar in all directions during the first half of the intervention (pre to mid, $\mathrm{F}(3,16)>5.0$, $p<0.0001$ ).

\subsection{Performance on an untrained task}

Since the star reaching task was performed 1024 times per session (960 assisted trials plus 64 unassisted trials), we examined performance on circle drawing. Circle drawing was performed only 20 times every two weeks. Figure 5 shows improvements in circularity of the drawn ellipses over the intervention. Circularity improved over the first half of the intervention $(\mathrm{F}(3,16)>4.0, p<0.0001)$. Beyond the midpoint of the intervention, there was no further improvement in circularity $(p>0.05)$.

\subsection{Changes in the motor map of the impaired hand}

Using single-pulse TMS, we examined the areal size and excitability (MEP size) of the motor map of the impaired hand. There was an increase in the area of the impaired FDI and ECR muscle motor map after therapy (Table 2 and Fig. 6) and six months later.

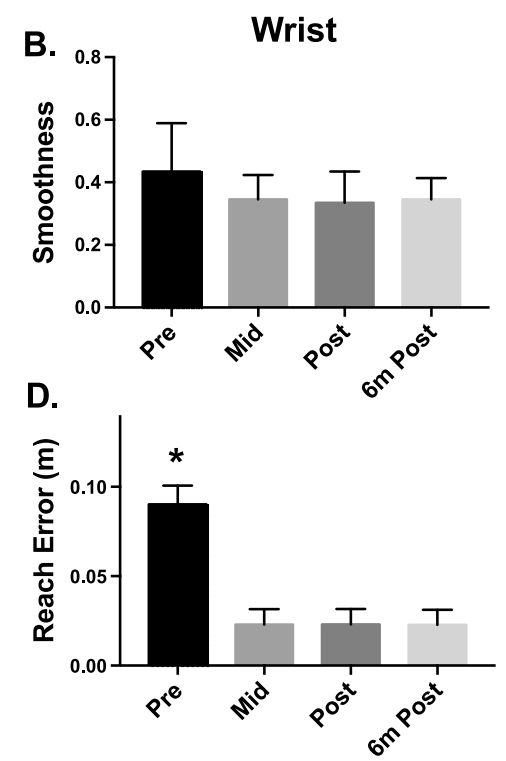

Fig. 2. Over the intervention, movement smoothness did not significantly change on the planar (A) or wrist (B) robots. Reach error on both the planar (C) and wrist (D) robots improved significantly from pre-intervention to the midpoint (week $6 ;{ }^{*} p<0.001$ ). There was no further improvement in reach error after the intervention or at the six-month follow-up. 

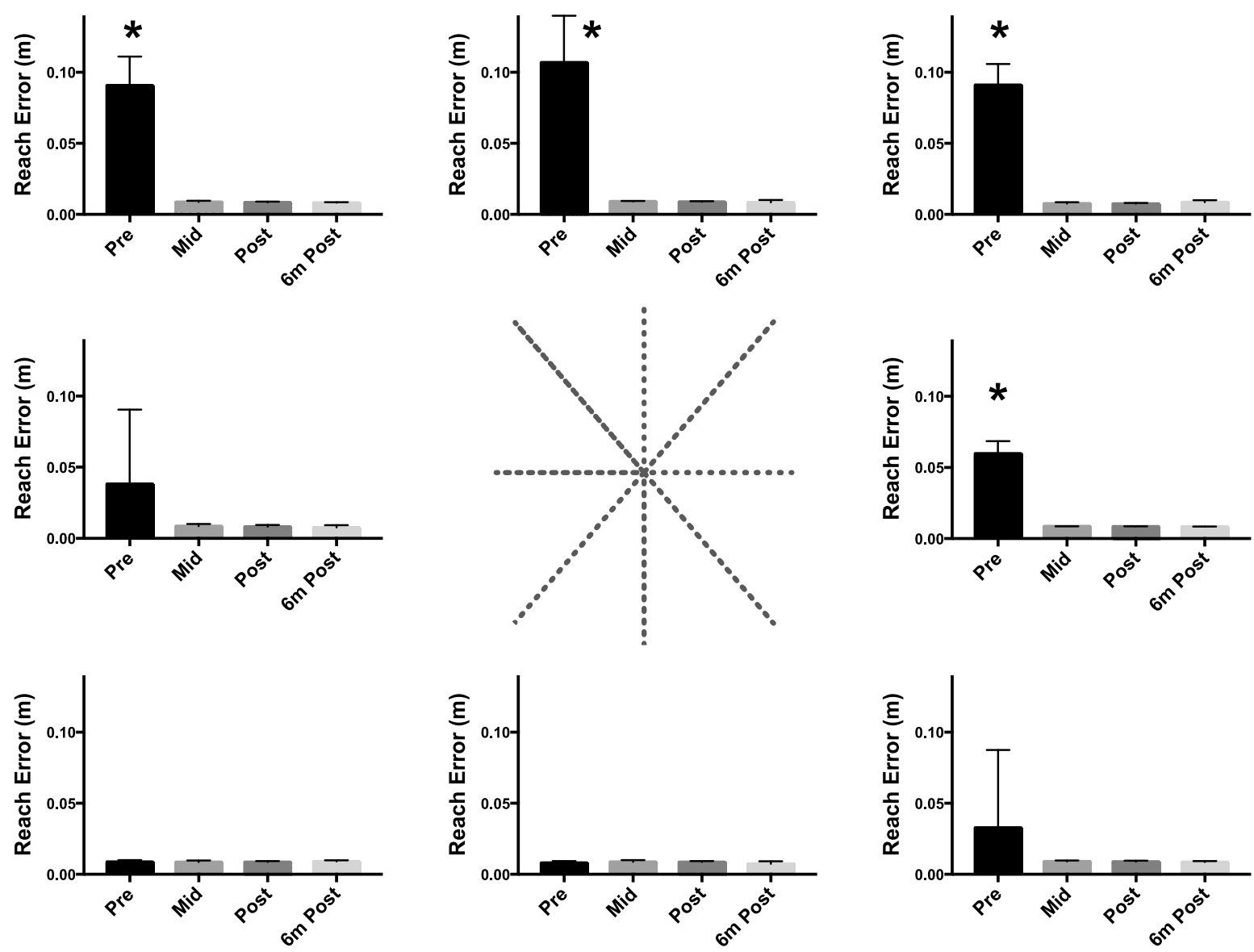

Fig. 3. Directional differences in planar reach error over the intervention. Each bar graph summarized reach error in the direction at which the graph is located relative to the center. The largest reach errors were found in the directions that required the participant to extend his upper limb. Error rates significantly decreased during the first half of the intervention $\left({ }^{*} p<0.01\right.$ compared to mid, post, and follow-up), and did not further improve during the second half of the intervention.

\subsection{Safety outcomes}

There were no adverse events in this study. Mild skin tingling and itching were reported after tDCS, and mild muscle soreness was reported after some robotic therapy sessions, particularly wrist robot therapy.

\section{Discussion}

In this pilot study, we aimed to determine safety and efficacy of combined tDCS and upper limb robotic therapy in an adult with USCP. This pilot study will inform future similar studies. We found that our innovative combination of excitatory tDCS and robotics was safe and produced long-lasting, clinically meaningful improvements in upper limb function.

\subsection{Efficacy of combined tDCS and robotic therapy}

The combination of $\mathrm{tDCS}$ and upper limb robotic therapy has been shown to improve motor outcomes in individuals with stroke (Yozbatiran et al., 2016) and spinal cord injury (Raithatha et al., 2016). Our findings indicate that this combination of technologies is also a feasible, effective intervention for adults with USCP. More work is needed to parse out the contributions of the tDCS and the robotic therapy to the recovery process, such that therapy can be provided in an optimal manner for each individual.

\subsection{Dosing of therapy}

We adopted our protocol from our work in stroke rehabilitation. We do not know if this dose and 

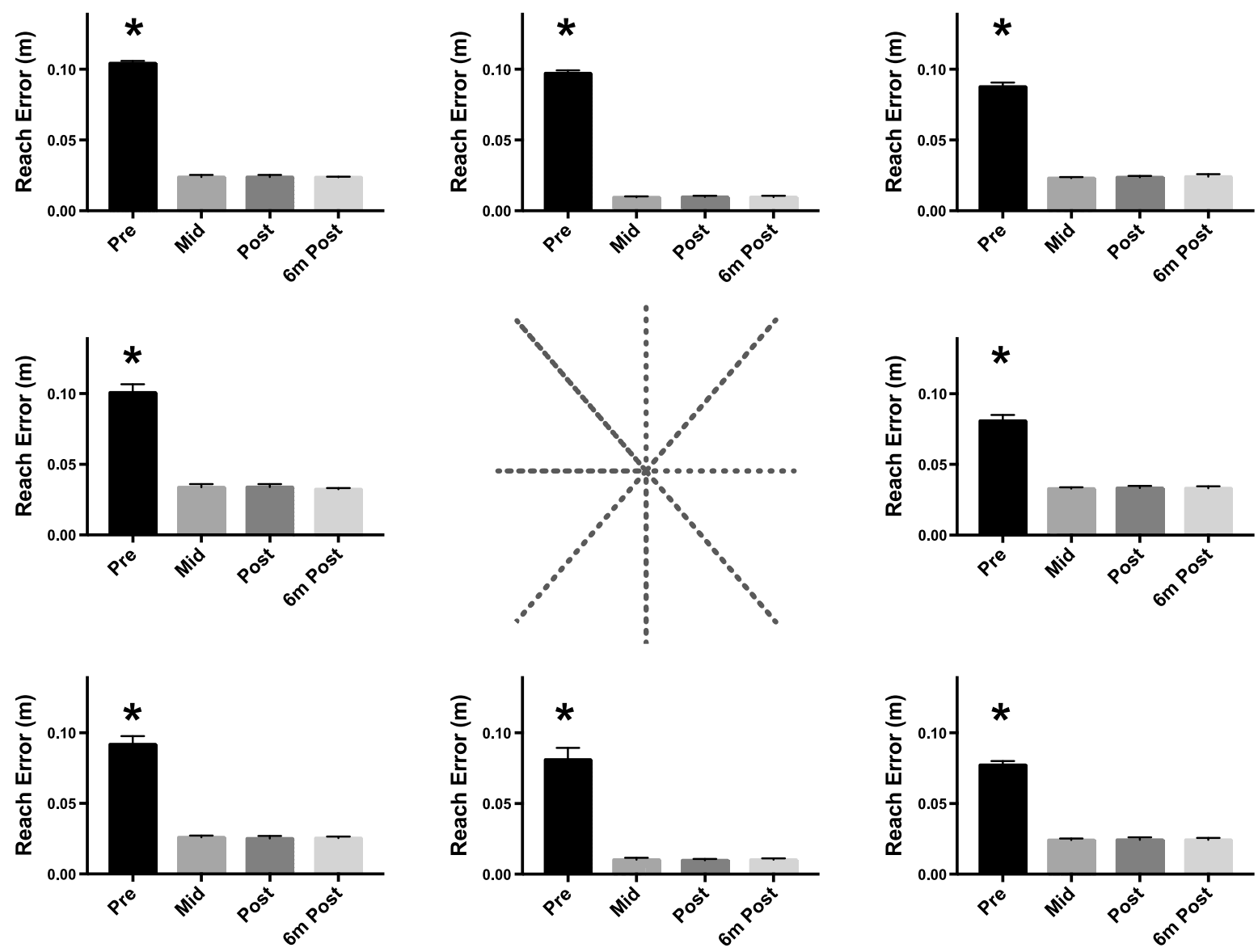

Fig. 4. Directional differences in wrist reach error over the intervention. Each bar graph summarized reach error in the direction at which the graph is located relative to the center. Reaching error in equal amounts were found in the first half of the intervention $\left({ }^{*} p<0.01\right.$ compared to mid, post, and follow-up), and did not further improve during the second half of the intervention.

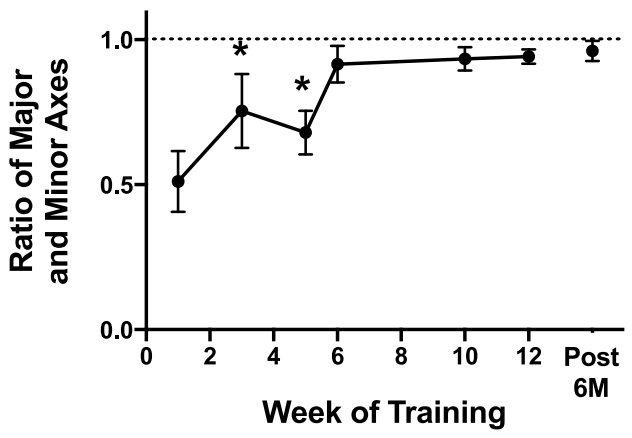

Fig. 5. Circularity of ellipses drawn on the planar robot. Twenty trials were performed every two weeks. The goal was to draw a perfect circle, whose ratio of major and minor axes would equal 1 (dotted line). During the half of the intervention, circularity improved compared to baseline $\left({ }^{*} p<0.001\right)$. No further significant improvements occurred during the second half of the intervention.

duration of therapy is ideal for people with USCP. Our findings in this participant indicate that he reached maximum accuracy on the robotic measures by the midpoint of the intervention period. Thus, for this individual, six weeks of therapy may have been sufficient to achieve optimal recovery. However, we do not know if the effects of therapy would have persisted for six months if the training period had been shorter. More work is needed to identify "stop signals" that indicate a participant has achieved their optimal improvements. The optimal dose is likely to vary among individuals, and a personalized approach is the ideal way to maximize each person's recovery. Indeed, Straudi et al. demonstrated that the efficacy of tDCS and upper limb robot-assisted therapy depended on the type of stroke and length of time since stroke, with individuals with chronic subcortical stroke showing better recovery than individuals with acute cortical stroke (Straudi et al., 2016). It is plausible that a longer duration of therapy may have boosted the recovery of individuals with acute cortical stroke. 
Table 2

Changes in neurophysiological measures after therapy

\begin{tabular}{lccc}
\hline TMS Measures & Before Therapy & After Therapy & Six Months After Therapy \\
\hline FDI map size $\left(\mathrm{cm}^{2}\right)$ & 27 & 34 & 25 \\
FDI MEP amplitude $(\mu \mathrm{V})(\mathrm{SD})$ & $95.6(53.4)$ & $120.9(64.5)$ & $191.8(110.6)$ \\
ECR map size $\left(\mathrm{cm}^{2}\right)$ & 19 & 25 & 31 \\
ECR MEP amplitude $(\mu \mathrm{V})(\mathrm{SD})$ & $211.6(54.8)$ & $148.0(66.3)$ & $241.7(48.7)$ \\
\hline
\end{tabular}
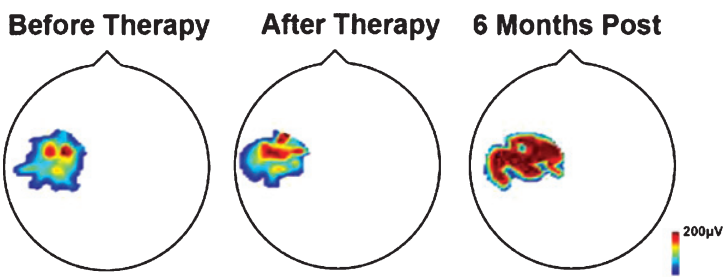

Fig. 6. Motor map of the participant's impaired FDI muscle. The heat map represents the amplitude of the MEP across locations of the map (blue = low amplitude, red = high amplitude). The FDI motor map increased in size and excitability after therapy.

\subsection{Therapy drives cortical plasticity}

Although this single case cannot be overinterpreted, our findings indicate that brain stimulation and skillful use of the impaired upper limb expands the motor map in adult CP. This finding is consistent with a robust body of literature that demonstrates expansions in cortical maps after skillful, repetitive use of the impaired limb (Friel et al., 2016; Jones et al., 1999; Liepert et al., 2000; Nudo, 2003; Sawaki et al., 2008).

\subsection{Individualized targeting of tDCS stimulation}

Although the clinical symptoms of USCP and stroke are similar, there are some key differences between adult-onset stroke and pediatric-onset USCP that is important to understand when translating a successful stroke therapy to USCP. In adult-onset stroke, the motor system has already achieved its mature, crossed pattern. In adult stroke, the loss of a contralateral pathway and reliance on an ipsilateral motor pathway to control the paretic side is thought to be a marker of severity and poor prognosis (Takeuchi et al., 2012). However, in USCP, developmental injury derails normal motor development. As many as $30 \%$ of people with USCP control the paretic side via ipsilateral motor pathways from the less-affected motor cortex (Staudt, 2007; Staudt et al., 2004). We have shown that these ipsilateral pathways have the potential to be functional and adaptive (Friel et al., 2016; Smorenburg et al., 2016).
Different studies have adopted different montages for tDCS application. In adult stroke, damage to one hemisphere causes downregulation of activity in that hemisphere and an imbalance in intracortical inhibition between the two hemispheres (Takechi et al., 2014). One commonly-used neuromodulation strategy in stroke is to suppress activity in the contralesional hemisphere, to decrease the inhibition of the ipsilesional hemisphere by the contralesional hemisphere (Zimerman et al., 2012). However, as described above, in people with $\mathrm{CP}$, developmental neuroplasticity often leads to reorganization of the motor system (Eyre et al., 2007). If the contralesional motor cortex controls movement of both upper limbs, suppression of activity in the contralesional hemisphere may not be of benefit. We propose a personalized approach: to target excitatory (anodal) tDCS to whichever hemisphere controls movement of the impaired upper limb. Importantly, in our pilot study, there was no decrement in skill of the lessimpaired upper limb (data not shown), indicating that tDCS over the less-affected M1 did not interfere with motor control of the less-impaired upper limb.

While our findings indicate that combined tDCS and robotic upper limb therapy was effective for improving motor function in an adult with $\mathrm{CP}$, it is necessary to study a larger number of individuals. We posit that evaluating clinical outcomes, kinematic improvements, and neurophysiological effects of the intervention will poise us to best understand the factors that contribute to recovery, the optimal therapy schedule and dose, and the optimal brain stimulation parameters. There is an urgent need for more effective therapies for adults with $\mathrm{CP}$, and we believe that combined tDCS and robotic therapy shows promise as a way to improve motor function and quality of life in adults with CP.

\section{Acknowledgments}

Funded by R03 HD084971. We are grateful to the participant for his involvement in this study. 


\section{Conflict of interest}

No authors have a conflict of interest with regards to this work.

\section{References}

Aarts, P. B., Jongerius, P. H., Geerdink, Y. A., van Limbeek, J., \& Geurts, A. C. (2010). Effectiveness of modified constraint-induced movement therapy in children with unilateral spastic cerebral palsy: A randomized controlled trial. Neurorehabil Neural Repair, 24(6), 509-518. doi: 10.1177/1545968309359767

Bosecker, C., Dipietro, L., Volpe, B., \& Krebs, H. I. (2010). Kinematic robot-based evaluation scales and clinical counterparts to measure upper limb motor performance in patients with chronic stroke. Neurorehabil Neural Repair, 24(1), 62-69. doi: $10.1177 / 1545968309343214$

Censor, N. (2013). Generalization of perceptual and motor learning: A causal link with memory encoding and consolidation? Neuroscience, 250, 201-207. doi: 10.1016/ j.neuroscience.2013.06.062

Dong, V. A., Tung, I. H., Siu, H. W., \& Fong, K. N. (2013). Studies comparing the efficacy of constraint-induced movement therapy and bimanual training in children with unilateral cerebral palsy: A systematic review. Dev Neurorehabil, 16(2), 133-143. doi: 10.3109/17518423.2012.702136

Edwards, D. F., Lang, C. E., Wagner, J. M., Birkenmeier, R., \& Dromerick, A. W. (2012). An evaluation of the Wolf Motor Function Test in motor trials early after stroke. Arch Phys Med Rehabil, 93(4), 660-668. doi: 10.1016/j.apmr.2011.10.005

Eyre, J. A., Smith, M., Dabydeen, L., Clowry, G. J., Petacchi, E., Battini, R., ...\& Cioni, G. (2007). Is hemiplegic cerebral palsy equivalent to amblyopia of the corticospinal system? Ann Neurol, 62(5), 493-503. doi: 10.1002/ana.21108

Friel, K. M., Kuo, H. C., Fuller, J., Ferre, C. L., Brandao, M., Carmel, J. B., .. \&\& Gordon, A. M. (2016). Skilled Bimanual Training Drives Motor Cortex Plasticity in Children With Unilateral Cerebral Palsy. Neurorehabil Neural Repair, 30(9), 834-844. doi: 10.1177/1545968315625838

Giacobbe, V., Krebs, H. I., Volpe, B. T., Pascual-Leone, A., Rykman, A., Zeiarati, G., ...\& Edwards, D. J. (2013). Transcranial direct current stimulation (tDCS) and robotic practice in chronic stroke: The dimension of timing. NeuroRehabilitation, 33(1), 49-56. doi: 10.3233/NRE-130927

Gladstone, D. J., Danells, C. J., \& Black, S. E. (2002). The fuglmeyer assessment of motor recovery after stroke: A critical review of its measurement properties. Neurorehabil Neural Repair, 16(3), 232-240.

Jones, T. A., Chu, C. J., Grande, L. A., \& Gregory, A. D. (1999). Motor skills training enhances lesion-induced structural plasticity in the motor cortex of adult rats. J Neurosci, 19(22), 10153-10163.

Kitago, T., \& Krakauer, J. W. (2013). Motor learning principles for neurorehabilitation. Handb Clin Neurol, 110, 93-103. doi: 10.1016/B978-0-444-52901-5.00008-3

Kleim, J. A., Kleim, E. D., \& Cramer, S. C. (2007). Systematic assessment of training-induced changes in corticospinal output to hand using frameless stereotaxic transcranial magnetic stimulation. Nat Protoc, 2(7), 1675-1684. doi: 10.1038/nprot.2007.206

Krebs, H. I., Hogan, N., Volpe, B. T., Aisen, M. L., Edelstein, L., \& Diels, C. (1999). Overview of clinical trials with MITMANUS: A robot-aided neuro-rehabilitation facility. Technol Health Care, 7(6), 419-423.

Liepert, J., Graef, S., Uhde, I., Leidner, O., \& Weiller, C. (2000). Training-induced changes of motor cortex representations in stroke patients. Acta Neurol Scand, 101(5), 321-326.

Liew, S. L., Santarnecchi, E., Buch, E. R., \& Cohen, L. G. (2014). Non-invasive brain stimulation in neurorehabilitation: Local and distant effects for motor recovery. Front Hum Neurosci, 8 , 378. doi: 10.3389/fnhum.2014.00378

Lin, K. C., Hsieh, Y. W., Wu, C. Y., Chen, C. L., Jang, Y., \& Liu, J. S. (2009). Minimal detectable change and clinically important difference of the Wolf Motor Function Test in stroke patients. Neurorehabil Neural Repair, 23(5), 429-434. doi: 10.1177/1545968308331144

Malouin, F., Pichard, L., Bonneau, C., Durand, A., \& Corriveau, D. (1994). Evaluating motor recovery early after stroke: Comparison of the Fugl-Meyer Assessment and the Motor Assessment Scale. Arch Phys Med Rehabil, 75(11), 1206-1212.

Morris, D. M., Uswatte, G., Crago, J. E., Cook, E. W. 3rd, \& Taub, E. (2001). The reliability of the wolf motor function test for assessing upper extremity function after stroke. Arch Phys Med Rehabil, 82(6), 750-755. doi: 10.1053/apmr.2001.23183

Norouzi-Gheidari, N., Archambault, P. S., \& Fung, J. (2012). Effects of robot-assisted therapy on stroke rehabilitation in upper limbs: Systematic review and meta-analysis of the literature. J Rehabil Res Dev, 49(4), 479-496.

Nudo, R. J. (2003). Functional and structural plasticity in motor cortex: Implications for stroke recovery. Phys Med Rehabil Clin N Am, 14(Suppl. 1), S57-76.

Page, S. J., Fulk, G. D., \& Boyne, P. (2012). Clinically important differences for the upper-extremity Fugl-Meyer Scale in people with minimal to moderate impairment due to chronic stroke. Phys Ther, 92(6), 791-798. doi: 10.2522/ptj.20110009

Paulus, W. (2004). Outlasting excitability shifts induced by direct current stimulation of the human brain. Suppl Clin Neurophysiol, 57, 708-714.

Raithatha, R., Carrico, C., Powell, E. S., Westgate, P. M., Chelette Ii, K. C., Lee, K., .. . \& Sawaki, L. (2016). Non-invasive brain stimulation and robot-assisted gait training after incomplete spinal cord injury: A randomized pilot study. NeuroRehabilitation, 38(1), 15-25. doi: 10.3233/NRE-151291

Sawaki, L., Butler, A. J., Leng, X., Wassenaar, P. A., Mohammad, Y. M., Blanton, S., ...\& Wittenberg, G. F. (2008). Constraint-induced movement therapy results in increased motor map area in subjects 3 to 9 months after stroke. Neurorehabil Neural Repair, 22(5), 505-513. doi: 22/5/505 [pii] $10.1177 / 1545968308317531$

Smorenburg, A. R., Gordon, A. M., Kuo, H. C., Ferre, C. L., Brandao, M., Bleyenheuft, Y., ...\& Friel, K. M. (2017). Does corticospinal tract connectivity influence the response to intensive bimanual therapy in children with unilateral cerebral palsy? Neurorehabil Neural Repair, 31(3), 250-260. doi: $10.1177 / 1545968316675427$

Staudt, M. (2007). (Re-)organization of the developing human brain following periventricular white matter lesions. Neurosci Biobehav Rev, 31(8), 1150-1156. doi: S0149-7634(07)000632 [pii] 10.1016/j.neubiorev.2007.05.005 
Staudt, M., Gerloff, C., Grodd, W., Holthausen, H., Niemann, G., \& Krageloh-Mann, I. (2004). Reorganization in congenital hemiparesis acquired at different gestational ages. Ann Neurol, 56(6), 854-863. doi: 10.1002/ana.20297

Straudi, S., Fregni, F., Martinuzzi, C., Pavarelli, C., Salvioli, S., \& Basaglia, N. (2016). tDCS and Robotics on Upper Limb Stroke Rehabilitation: Effect Modification by Stroke Duration and Type of Stroke. Biomed Res Int, 2016, 5068127. doi: $10.1155 / 2016 / 5068127$

Takechi, U., Matsunaga, K., Nakanishi, R., Yamanaga, H., Murayama, N., Mafune, K., \& Tsuji, S. (2014). Longitudinal changes of motor cortical excitability and transcallosal inhibition after subcortical stroke. Clin Neurophysiol, 125(10), 2055-2069. doi: 10.1016/j.clinph.2014.01.034

Takeuchi, N., \& Izumi, S. (2012). Maladaptive plasticity for motor recovery after stroke: Mechanisms and approaches. Neural Plast, 2012, 359728. doi: 10.1155/2012/359728

Turner, D. L., Ramos-Murguialday, A., Birbaumer, N., Hoffmann, U., \& Luft, A. (2013). Neurophysiology of robotmediated training and therapy: A perspective for future use in clinical populations. Front Neurol, 4, 184. doi: 10.3389/fneur.2013.00184

Volpe, B. T., Huerta, P. T., Zipse, J. L., Rykman, A., Edwards, D., Dipietro, L., ...\& Krebs, H. I. (2009). Robotic devices as therapeutic and diagnostic tools for stroke recovery. Arch Neurol, 66(9), 1086-1090. doi: 10.1001/archneurol.2009.182

Yozbatiran, N., Keser, Z., Davis, M., Stampas, A., O’Malley, M. K., Cooper-Hay, C., .. \& \& Francisco, G. E. (2016). Transcranial direct current stimulation (tDCS) of the primary motor cortex and robot-assisted arm training in chronic incomplete cervical spinal cord injury: A proof of concept sham-randomized clinical study. NeuroRehabilitation, 39(3), 401-411. doi: 10.3233/NRE-161371

Zimerman, M., Heise, K. F., Hoppe, J., Cohen, L. G., Gerloff, C., \& Hummel, F. C. (2012). Modulation of training by single-session transcranial direct current stimulation to the intact motor cortex enhances motor skill acquisition of the paretic hand. Stroke, 43(8), 2185-2191. doi: 10.1161/ STROKEAHA.111.645382 